\title{
Age Related Changes in Physical Activity and Incidence of Non-Communicable Diseases in a Sample of Kuwaiti Adults
}

\author{
Nadia Garashi, Jasem Ramadan Al-Kandari, Mario Barac Nieto* \\ Department of Physiology, Health Sciences Center, Kuwait University, Jabriya, Kuwait \\ Email: *mario.baracnieto@gmail.com
}

How to cite this paper: Garashi, N., Al-Kandari, J.R. and Nieto, M.B. (2021) Age Related Changes in Physical Activity and Incidence of Non-Communicable Diseases in a Sample of Kuwaiti Adults. Health, 13, 505-525.

https://doi.org/10.4236/health.2021.135039

Received: March 30, 2021

Accepted: May 11, 2021

Published: May 14, 2021

Copyright $\odot 2021$ by author(s) and Scientific Research Publishing Inc. This work is licensed under the Creative Commons Attribution International License (CC BY 4.0).

http://creativecommons.org/licenses/by/4.0/

\begin{abstract}
The incidence of major chronic Non Communicable Diseases (NCD) medically diagnosed or treated, was studies in a cross section of Kuwait adult population as well as their daily levels of physical activity (PA) as recalled by the International Physical Activity Questionnaire, IPAQ. A group of 1957 subjects 17 - 65 years of age, representative of the Kuwait population was selected using last year high school students and their relatives as well as government workers in each Kuwait governorate. Descriptive statistics, frequency tables, chi square and Fisher tests were used. ANOVA or Mann Whitney and Kruskal-Wallis tests were used for comparisons. The percentage of subjects in the low categorical PA level (from IPAQ) is high (29.4\%) even at a young age (17 - 24), similar in prevalence to overweight/obesity (30\%). At age 45, obesity increases to $40 \%$ with little change in \% subjects of low PA. Weighted average categorical PA was 1280 MET.min/week reflecting that the majority (40\%) of subjects belonged to the moderate PA category. A high proportion (38\%) of subjects in the $17-24$ age category contributed to the observed level of PA activity. Incidence of overweight and obesity increased from 30 to more than $55 \%$ from the second to the sixth decade of life. Hyperlipidemias increased in incidence from $8 \%$ to $45 \%$ of the sample from the third to the sixth decades of age. Hypertension and diabetes increased from the $4^{\text {th }}$ to the $6^{\text {th }}$ decades of life to affect from $14 \%$ to $40 \%$ and from $10 \%$ to $36 \%$ of the studied population, respectively. Incidence of Heart Disease increased from $9 \%$ to $15 \%$ of the sample population, between the $5^{\text {th }}$ and $6^{\text {th }}$ decades of life. The data indicate a sequence of events, initiated by overweight and obesity as early as in the $2^{\mathrm{d}}$ decade of life, followed by hyperlipidemia in the $3^{\mathrm{d}}$ decade, diabetes and hypertension in the $4^{\text {th }}$, and heart disease in the $5^{\text {th }}$ decade of life. Changes in incidence of low energy expenditure (low PA) were not associated with the observed changes in prevalence of obesity/overweight with age
\end{abstract}


which are likely due to increments in energy intake in groups with insufficient PA. Changes in low PA incidence with age did not correlate with those in NCD. Interventions to minimize overweight and hyperlipidemia should be started in the second and third decades of life respectively. Increases in PA may potentiate the responses to these treatments.

\section{Keywords}

Daily Physical Activity, IPAQ, Recall, Survey, Kuwait, Cardiovascular, Diabetes, Hypertension, Overweight, Obesity, Hyperlipidemia, Age, Adults

\section{Introduction}

The changes in physical activity (PA) and in the incidence of major chronic diseases were investigated in a cross sectional sample of 1957 adult subjects, representative of the Kuwaiti population and whom responded to a previously [1] test-retest and objectively validated questionnaire in which weekly episodes of PA and specific chronic diseases' medical diagnoses or treatments were recalled.

\subsection{Effect of PA on Health and Disease}

PA has major beneficial effects on most chronic diseases. These benefits are not limited to preventing or limiting the progression of disease, but include improving physical fitness, muscular strength and the quality of life [2]. In older people, regular physical activity increases the potential for independent living. WHO recently reviewed the evidence for the health effects of PA [3]. In summary:

\subsubsection{Cardiovascular Disease}

Inactive people have up to twice the risk of heart disease compared to active people. PA also helps to prevent stroke [4], and improves risk factors including high cholesterol [4] high blood pressure, and cardiac performance [5].

\subsubsection{Overweight and Obesity}

Low levels of PA are a significant factor in the dramatic increase in obesity prevalence in many regions. Body weight normally increases with age, but habitual, lifetime PA can reduce weight gain. Participation in appropriate amounts of activity can support healthy weight maintenance or even weight loss [6]. PA is also important to reduce weight in people who are overweight or obese [7].

\subsubsection{Diabetes}

Diabetes is an increasing medical concern, as rates of type 2 (non-insulindependent) diabetes increase. Type 2 diabetes typically occurs in adults aged over 40, although cases are emerging among children and young people as obesity rates rise [8].

Strong evidence indicates that PA helps to prevent type 2 diabetes; the risk for active people is about $30 \%$ lower than that for inactive people [9]. Both mod- 
erate- and vigorous-intensity PA reduce the risk [10] [11], but PA must be done regularly.

\subsubsection{Cancer}

$\mathrm{PA}$ is associated with a reduction in the overall risk of cancer. There is a protective effect of PA on the risk of colon cancer [12]; the risk for active people is around $40 \%$ lower. PA is also associated with a reduced risk of breast cancer among postmenopausal women and improved survival [13] and evidence shows that vigorous PA may provide a protective effect against prostate cancer [14].

\subsubsection{Musculoskeletal Health}

Participation in PA throughout life can increase and maintain musculoskeletal health, or reduce the decline that usually occurs with age in sedentary people [15]. Participation by older adults can help maintain strength and flexibility, helping older people to perform daily activities [15] and reduce risk of falls and hip fractures. Participation in weight-bearing activities (jumping or skipping) helps increase bone density and prevents osteoporosis [16]. This is particularly important for adolescents and menopausal women.

\subsection{Assessment of PA}

The recall questionnaire is the most readily available instrument for assessing PA in large groups or populations. There are many such questionnaires, such as the Finbalt Health Monitor [17], Behavioral Risk Factor Surveillance State (BRFSS) Questionnaire [18], Canadian PA Monitor [19], IPAQ [20], and Global PA Questionnaire (GPAQ) [21], among others. However, many of the existing instruments are not comparable in the type of activities surveyed (i.e., frequently only limited to leisure-time activities) and differ in their formats for data collection.

\subsubsection{International Physical Activity Questionnaire, IPAQ}

The IPAQ instruments underwent several stages of development and testing, culminating in a large multi-country reliability and validity study, carried out in 14 centers in 12 countries across 6 continents during the year 2000. The final results demonstrated that IPAQ instruments have acceptable measurement properties for use in many settings and in different languages and are also suitable for monitoring PA at the population level in diverse settings. It has measurement properties that are at least as good as other established self-report PA measures. Thus it provides internationally comparable data on health-related PA [20].

IPAQ has a short form, recommended for national monitoring, and a long form for research purposes and for intervention planning. The reference time period is the last 7 days. There are two formats: self-administered and telephone or face-to-face interview.

IPAQ is not limited to leisure time activities but assesses PA undertaken across a comprehensive set of domains.

In the IPAQ short form, the specific types of activity that are assessed are 
walking, moderate-intensity activities (defined here as PA that produce a moderate increase in respiration rate, heart rate and sweating of at least 10 minute duration) and vigorous-intensity activities (defined as PA producing vigorous increases in respiration rate, heart rate and sweating for at least 10 min duration). IPAQ also includes questions about time spent sitting/lying as an indicator of sedentary behavior.

The items in the short IPAQ form were structured to provide separate scores on walking, moderate-intensity and vigorous-intensity activity. Computation of the total score for the short form requires summation of the duration (in minutes) and frequency (days) of walking, moderate-intensity and vigorous-intensity activities in each subject. Domain-specific estimates are not defined.

\subsubsection{Validation of IPAQ}

We studied the reliability and objective validity of an Arabic version of IPAQ in Kuwait [1], showing:

1) Objective measures of daily PA in Arabic subjects for the first time.

2) Quantitative estimates of daily PA using the IDEEA activity meter in high and moderate PA groups. In the low activity group IDEEA overestimated the activity.

3) Daily PA was estimated by the IPAQ short form (IPAQ-SF) recalling frequency and duration of bouts of walking, and of PA with moderate or heavy breathing and sweating, during one week, followed by MET conversions.

4) Average EE (energy expenditure) in subjects with high activity levels was significantly different from those with low activity levels. However, average EE in moderately active subjects did not significantly differ from that in sedentary subjects, pointing to too lax criteria used in the definition of moderate activities (often confused with leisure walking) and to high variance of the data.

5) Good (0.69) correlation between IPAQ-SF and objective IDEEA measurements of EE.

6) Both IDEEA and IPAQ detected higher EE in subjects with normal BMI than in those with high BMI.

7) Higher EE in males than in females disappears when expressed per unit Body weight.

\subsubsection{Review of PA Data Derived from IPAQ and Other Tools}

IPAQ allows a categorical score denoting one of three PA levels (Low, Moderate or High) according to criteria related to the time (in minutes and days), and intensity in MET-min per week (see Table 1).

In Sweden [22] the percentage of the sample falling in low, moderate, and high categorical PA levels were 37\%, 37\%, and 26\%; respectively. In Saudi Arabia [23] most, $40.6 \%$ were inactive (low PA category), $34.3 \%$ were moderately active and only $25.1 \%$ met so-called health-enhancing levels of PA.

Increasingly, the lack of PA is associated with increased mortality and morbidity in the USA [24], in the European Union [25] and using data from 
Table 1. Percentage of PA categories based on corresponding criteria.

\begin{tabular}{|c|c|c|c|c|c|}
\hline \multirow{2}{*}{$\begin{array}{l}\text { Categorical } \\
\text { PA level }\end{array}$} & \multirow{2}{*}{ Sub-type } & \multirow{2}{*}{ IPAQ categorical PA level criteria } & \multicolumn{3}{|c|}{ Percentage } \\
\hline & & & Males & Females & All \\
\hline \multirow[b]{2}{*}{ 1) Low } & 1.1 & No activity is reported & $7.8 \% \mathrm{a}$ & $8.7 \% \mathrm{~b}$ & $8.3 \%[0]$ \\
\hline & 1.2 & $\begin{array}{l}\text { Some activity is reported but not enough to } \\
\text { meet moderate or high categories }\end{array}$ & $19.3 \% a$ & $22.3 \%$ b & $\begin{array}{r}21.1 \% \\
\{500\} \\
{[105]}\end{array}$ \\
\hline \multirow{3}{*}{ 2) Moderate } & 2.1 & $\begin{array}{l}3 \text { or more days of vigorous activity of at least } \\
\qquad 20 \text { minutes per day }\end{array}$ & $6.4 \% \mathrm{a}$ & $3.7 \% \mathrm{~b}$ & $\begin{array}{c}4.8 \% \\
\{640\} \\
{[31]}\end{array}$ \\
\hline & 2.2 & $\begin{array}{l}5 \text { or more days of moderate-intensity activity } \\
\text { and/or walking of at least } 30 \text { minutes per day }\end{array}$ & $22.4 \% \mathrm{a}$ & $31.9 \% \mathrm{~b}$ & $\begin{array}{c}28 \% \\
\{1200\} \\
{[336]}\end{array}$ \\
\hline & 2.3 & $\begin{array}{c}5 \text { or more days of any combination of walking, } \\
\text { moderate-intensity or vigorous-intensity } \\
\text { activities achieving a minimum of at least } 600 \\
\text { MET-minutes / week }\end{array}$ & $5.5 \% \mathrm{a}$ & $3.1 \% \mathrm{~b}$ & $\begin{array}{c}4.1 \% \\
\{1440\} \\
{[59]}\end{array}$ \\
\hline \multirow{2}{*}{ 3) High } & 3.1 & $\begin{array}{c}\text { Vigorous-intensity activity on at least } 3 \text { days } \\
\text { and accumulating at least } 1500 \\
\text { MET-minutes/week }\end{array}$ & $26.4 \%$ a & $11.3 \% \mathrm{~b}$ & $\begin{array}{c}17.5 \% \\
\{1500\} \\
{[263]}\end{array}$ \\
\hline & 3.2 & $\begin{array}{c}\text { Any combination of walking, moderate- or } \\
\text { vigorous-intensity activities accumulating at } \\
\text { least } 1500 \text { MET-minutes/week }\end{array}$ & $12.2 \% \mathrm{a}$ & $19.1 \% \mathrm{~b}$ & $\begin{array}{c}16.2 \% \\
\{3000\} \\
{[486]}\end{array}$ \\
\hline
\end{tabular}

$\mathrm{X}^{2}=105.5 ; \mathrm{p}<0.001$. all groups [1280]. \{ \} MET.min/w for subgroup, [ ] \% Weighed MET.min/w contribution to whole group EE PA.

cellphone apps, in many countries throughout the world [26].

\section{Methods}

\subsection{Permission, Consents, and Confidentiality}

The project was granted permission from the Department of Physiology and the Faculty of Medicine at Kuwait University and obtained approval of the Ethics Board Commission of the Faculty. Permission was also secured from the Ministry of Education to administer the questionnaire in selected public schools. Consent was obtained after explaining aim, procedure, precautions, and freedom of withdrawal. Moreover, confidentiality was assured for participants all throughout the study.

\subsection{Study Population}

This study targets Kuwaiti citizens from both genders belonging to the age group 17 - 65 years and are able to perform all types of PA. Criteria for inclusion or exclusion are described below (Section 2.5).

\subsection{Sampling}

Sampling is necessary when it is difficult logistically to survey the whole population. For sampling to be representative and generalizable, it has to be random. 
The stratified sampling technique was used in the survey phase for the selection of the settings and the subjects. For each governorate (major country subdivisions), and with the help of Ministry of Education, sample frames were prepared for all public secondary schools (males and females), and major public service centers. In addition, similar sample frames were prepared for private public schools nation-wide and various governmental ministries and departments housed at the complex of ministries located in Kuwait City.

Regarding subjects from schools, 2 high schools or more (for each gender) were randomly selected from each governorate. However, if 2 secondary schools (same or different sexes) were located in the same residential area, one was randomly omitted and another was chosen randomly. Within each school, classes were randomly chosen among science or art branches. All available students (meeting criteria of inclusions) were chosen within each selected class. Self-administered questionnaires were then distributed among students, so that one of their household members in the age group 17 to 65 years was to fill the questionnaire and then return it to the school for the data-collectors. To ensure that the household member filling the questionnaire is randomly chosen, the Kish technique was used, where only the person with the nearest birth date to the date of questionnaire distribution was chosen. Students in the target age group (i.e. 17 or older) could also fill the questionnaire if their birth date was the nearest.

In addition, 2 private schools were randomly chosen from the sample frame. Subsequently, similar procedure was followed in the administration of the questionnaire.

Regarding subjects from work places, a number of government departments were randomly chosen from the aforementioned sample frame and all available employees meeting the inclusion criteria were approached.

\subsection{Sample Size}

The size of the sample for the population survey was derived using Equation (1):

$$
n=z^{2} \times p \times q / d^{2}
$$

where:

$Z=1.96$, for $95 \%$ Confidence Interval (CI), type I error is $\alpha=0.05 ; Z \alpha=1.96$ (two-sided),

$p=$ Proportion of target population. The calculated proportion of the target population (Kuwaitis aged 17 to 65 years) to the total population of Kuwait is 0.51 ,

$q=(1-p)=$ Proportion of those who do not belong to target population equals 0.49 ,

$d=$ desired level of precision, or maximum \% difference we are willing to accept between true population rate and sample rate. Two \% was chosen, so $d=$ 0.02 , 


$$
\text { Thus } n=1.96^{2} \times(0.51 \times 0.49) / 0.02^{2}=2327
$$

In summary, assuming $95 \%$ confidence, and precision of 0.02 (which also presumes heterogeneity of the population), the sample size for the large-scale survey was calculated to be roughly 2400 subjects.

\subsection{Criteria for Inclusion or Exclusion}

Subjects should be Kuwaiti citizens, speak Arabic, aged between 17 and 65 years and free of locomotor handicap. Exclusion criteria include Non-Kuwaitis, and Kuwaitis younger than 17 years or older than 65 years, or not available during the study period, or suffering from a locomotor handicap.

\subsection{KPAQ and IPAQ}

"IPAQ" refers to the 7-item, short-form, and self-administered format of the IPAQ questionnaire. KPAQ, on the other hand, encompasses "IPAQ-SF," in addition to anthropometric demographic and health history related items. The "Questionnaire" includes also a separate instructions sheet.

\subsubsection{Parts and Format of KPAQ Questionnaire}

KPAQ refers to collection of elements, in which IPAQ is the PA measuring tool. However, there are other important components. Like IPAQ, KPAQ is a self-administered recall instrument. It is composed of 44 items with varying length and complexity. The 7 IPAQ items are presented first. Other items include demographic information, health history and status, patterns of PA/exercise, Arabic and English-translations of KPAQ are available on demand.

\subsubsection{Instruction Sheet}

The instruction sheet was attached to the envelope in which each questionnaire was placed during this Survey. It served to Inform subjects about 1) the inclusion criteria (Kuwaiti nationality, age range, ambulant condition; 2) Encourage reading of the questionnaire's introduction, questions and options thoroughly; 3) Remind subjects that incomplete or hasty answers might render the data inaccurate, or useless for the analysis. This included the unduly hasty choice of "not sure/I don't know" options provided in the IPAQ section of the questionnaire. 4) Emphasize some of the key concepts in IPAQ such as the 10 minute duration of PA bouts, and multiple PA domains that should be taken in account while answering IPAQ questions.

\subsubsection{Patterns of PA/Exercise}

Subjects are asked whether they perform regular PA or exercise. If they do, they are given a list of exercises to tick (more than one tick is permitted): Swedish exercises (calisthenics), walking, jogging, running, soccer, basketball, volleyball, swimming/diving, weight-lifting, aerobics, dancing and other sports were listed.

\subsubsection{Demographics}

One page of the questionnaire collects demographic information which includes: 
- Gender.

- Age.

- Height in centimeters.

- Weight in kilograms.

- Marital Status: whether married, single, or other (divorced, separated, widowed).

- Employment Status: whether student, working, or not working. Retired subjects were expected to choose the option "not working," unless they were involved in any commercial or volunteering activity, where they could choose "working."

- Average Daily Working Hours (for students: attendance time).

- Level of PA encountered at work: 3 options were given:

o Overall low-intensity (normal breathing)

o Involving moderate-PA (breathing somewhat higher than normal)

o Involving vigorous-PA (breathing much higher than normal)

- Education Level: namely, highest degree attained. Options were:

o Illiterate

o Less than high school (includes high school students)

o High school graduate/diploma/college student

o University degree and beyond

- Housing: whether in house or apartment.

- Governorate in their Civil IDs or that to where they have now moved.

\subsubsection{Health History and Status Questions}

The subject is asked a series of questions answered by yes or no, pertaining to whether he/she was diagnosed, or is on treatment for a list of illnesses: heart disease, diabetes, hypertension, hyperlipidemia, osteoporosis, cancer or others.

Questions about overweight or obesity had a similar format, and subjects were also asked about their self-perception.

Smoking subjects are asked about number of cigarettes consumed per day and years of smoking. Hubble-bubble smokers are asked about frequency per day, per week, and duration (in minutes) of each session, as well as years of this type of smoking.

\subsection{Data Analyses}

Data entry and cleaning was performed using Office Excel 2003 software (Microsoft, Washington, USA). The former software was also used to compute some variables and to sum up data to produce averages (e.g. total EE in MET·min/w), but statistical analysis was mainly performed using SPSS 16 software (IBM, Armonk, NY, USA).

\subsubsection{Data Derived from KPAQ/IPAQ}

Categorical PA level score, where subjects were classified into one of three levels of PA: Low, moderate, and high PA level, according to IPAQ categorical scoring 
criteria (Table 1).

\subsubsection{Statistics}

Descriptive analysis was performed for all variables, showing Means and standard error of the mean (SEM) and/or coefficient of variation (CV) for normally distributed values (normality detected using Kolmogorov Smirnov test), or medians and quartiles otherwise. Frequency tables were created for categorical variables. For comparisons and associations of categorical variables, cross-tabulation with chi-square maximum likelihood and the exact probability Fisher tests were used. For continuous variables, ANOVA and t-tests were used in normally distributed data; the Mann-Whitney U test and the Kruskal-Wallis tests were used for nonparametric data, as indicated in the text.

\section{Results}

\subsection{Demographics}

A total of 1957 questionnaires were included. Age was found to be normally distributed (Kolmogorov-Smirnov $\mathrm{z}$ test). Mean age is 33.2 years and standard deviation is 13.2 years. Median age is 33 years. Females comprised $59 \%$ of the sample, higher than that in the corresponding Kuwait population. There was no significant difference in age between males and females.

Most subjects were young (ages 17 - 24), representing about 38\% of the sample and much greater than the $12.5 \%$ that this age group represents in the corresponding Kuwait population (Figure 1). Married subjects accounted for $57.7 \%$ of the sample, while $37.1 \%$ were single. The remaining $5.2 \%$ were divorced, widowed or separated. Most subjects (46.9\%) had at least some high school or high school diploma but $27.1 \%$ had no high school education. About a quarter

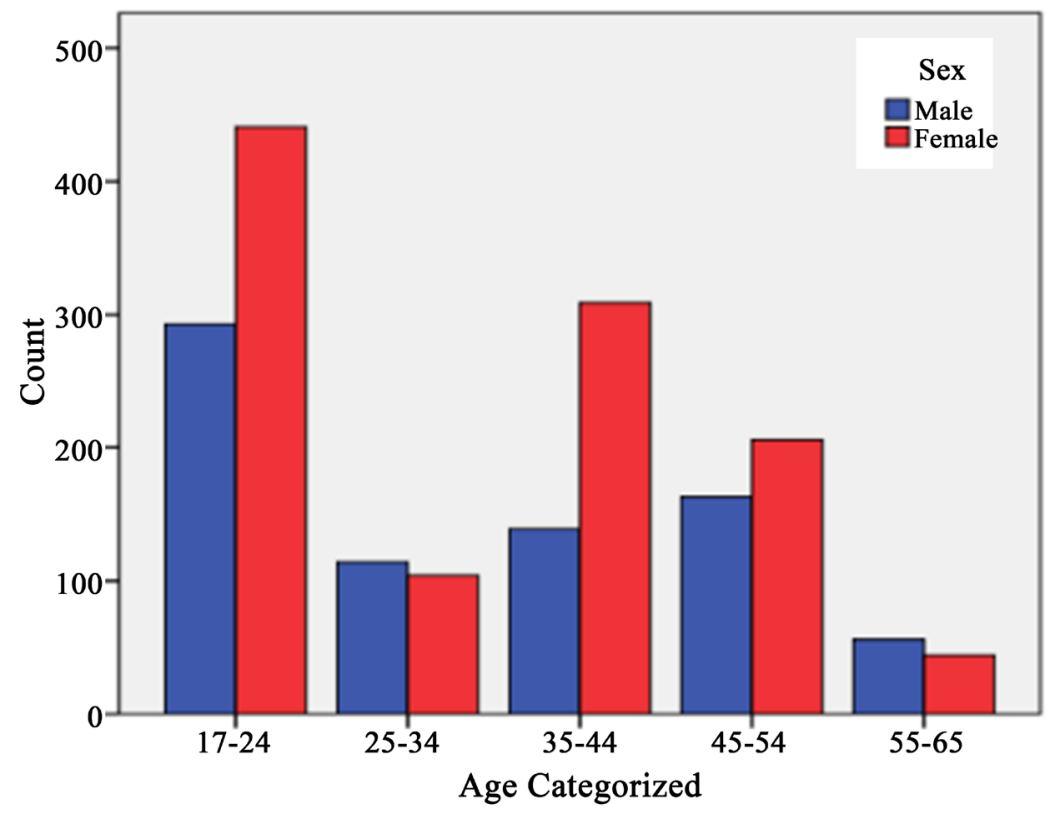

Figure 1. Age distribution in males (blue) and females (red). 
(24.9\%) had University education. Only $1 \%$ were illiterate.

Roughly half of the sample (49.9\%) was employed (working). Students comprised $30.8 \%$ of total. A minority was not working (19.3\%). More females were not working (e.g. housework, 23\%) compared to males $(9.3 \%)(\mathrm{p}<0.001)$. The daily duration of occupation was 6.6 hours (median 7 hours; normally distributed according to Kolmogorov-Smirnov test). On average, males worked slightly longer hours (6.72 hours) than females (6.45 hours) $(\mathrm{p}<0.005)$.

Only $6.1 \%$ perceived their work as vigorous PA. For another $36.1 \%$, their work involved moderate PA. The remaining $57.8 \%$ had only low PA at work. Of the 399 missing cases, 376 subjects were non-workers/non-students, and 23 chose not to answer.

Predictably, mean height (Table 2) for males $(172 \mathrm{~cm})$ is higher than that of females $(159.9 \mathrm{~cm})(\mathrm{p}<0.001)$. Similarly, mean weight in males $(80.3 \mathrm{~kg})$ is higher than that in females $(69.7 \mathrm{~kg})(\mathrm{p}<0.001)$. Nevertheless, mean BMI is not different in males compared to females $\left(27.2 \mathrm{~kg} / \mathrm{m}^{2}\right)$.

When BMI was categorized (according to WHO classification) the Normal BMI group accounted for $37.7 \%$, those with Overweight for $34.1 \%$, and the obese for $28.2 \%$ of the group. There were no significant differences between males and females in BMI category distribution $(\mathrm{p}>0.3)$. The percentages of the sample who were overweight (BMI $\geq 25 \mathrm{~kg} / \mathrm{m}^{2}$ but $<30 \mathrm{~kg} / \mathrm{m}^{2}$ ) or obese (BMI $\geq 30$ $\mathrm{kg} / \mathrm{m}^{2}$ ) were each plotted across age categories A significant increase is noted for both as age increases, overweight status reaches a plateau at age group $35-44$. Obesity, however, continues a significant rise till age group 45 - 54 .

\subsection{IPAQ PA Results}

Median sitting-time was 300 minutes/day (5 hours/day). Medians of sitting time showed no difference across sex, age categories, marital status, and employment status.

Subjects who do moderate or vigorous PA at work reported significantly ( $\mathrm{p}<$ 0.001) lower siting time than the less active subjects, Sitting time also increased significantly in direct relationship with education status.

\subsection{Categorical PA Level Scores}

All cases fall into one of the following 3 levels of PA: low, moderate and high, based on an algorithm encompassing most of the IPAQ variables. Table 1 shows the percentages of categorical PA levels according to the criteria met. More than

Table 2. Mean weight, height, and BMI for males and females.

\begin{tabular}{cccccc}
\hline & \multicolumn{2}{c}{ Height $(\mathrm{cm})$} & \multicolumn{2}{c}{ Weight $(\mathrm{kg})$} & BMI $\left(\mathrm{kg} / \mathrm{m}^{2}\right)$ \\
\cline { 2 - 6 } & Mean & $(95 \% \mathrm{CI})$ & Mean & $(95 \% \mathrm{CI})$ & Mean \\
\hline Males $(\mathrm{n}=741)$ & 172 & $(171.4-172.6)$ & 80.3 & $(79.1-81.6)$ & 27.2 \\
Females $(\mathrm{n}=1041)$ & 159.9 & $(159.5-160.3)$ & 69.7 & $(68.7-70.6)$ & 27.2 \\
Total $(\mathrm{n}=1782)$ & 164.9 & $(164.5-165.4)$ & 74.1 & $(73.3-74.9)$ & 27.2 \\
\hline
\end{tabular}


$2 / 3$ of subjects with moderate PA level met the criteria with a combination of moderate and walking PA, rather than vigorous PA, especially among females. In fact, females were less prone to vigorous PA but some still reached moderate or high PA categories levels. Males on the other hand, met criteria of high PA level more commonly based on performing vigorous PA. All these findings were statistically significant $(\mathrm{p}<0.05)$.

Overall, $33.7 \%$ of the cases satisfy the criteria for high categorical PA level, (Table 1) more in males (38.6\%) than in females (30.3\%). Most females in the high PA category engaged in vigorous activities reaching 1500 MET.min/w. A fewer percentage of females engaged in combinations of vigorous or moderate activity and walking. Most males combined vigorous activity and walking and fewer did only vigorous PA. Overall, (Table 1) subjects combining vigorous with other activities spent on average about $3000 \mathrm{MET}$.min/week (such as $300 \mathrm{~min} / \mathrm{w}$ at $8 \mathrm{MET}$ and $150 \mathrm{~min} / \mathrm{w}$ at $4 \mathrm{METS}$ ). Most subjects, about 37\%, were moderately active and fewer (29.4\%) were of low PA. Females were more likely to fall in the moderate PA level.

Figure 2 shows percentages of the 3 categorical PA levels at various decades of age. The high PA level category was most prevalent at younger ages (i.e. 17-24 and 25 - 34 years) where it represented $39.5 \%$ and $37.2 \%$ of subjects respectively. In contrast, the group in the $45-54$ years of age had the lowest proportion of subjects at the high PA level $(\mathrm{p}<0.05)$. In fact, $59 \%$ of all high PA level cases were aged 34 years or younger. Subjects older than 34 were more likely to fall in the moderate PA level $(\mathrm{p}<0.05)$. Furthermore, the $\%$ of subjects in the low PA category was highest (34\%) after 45 years of age and was smallest $(27.8 \%)$ in the youngest group (17 - 24 years).

Low PA level was least prevalent among those with normal BMI, and highest

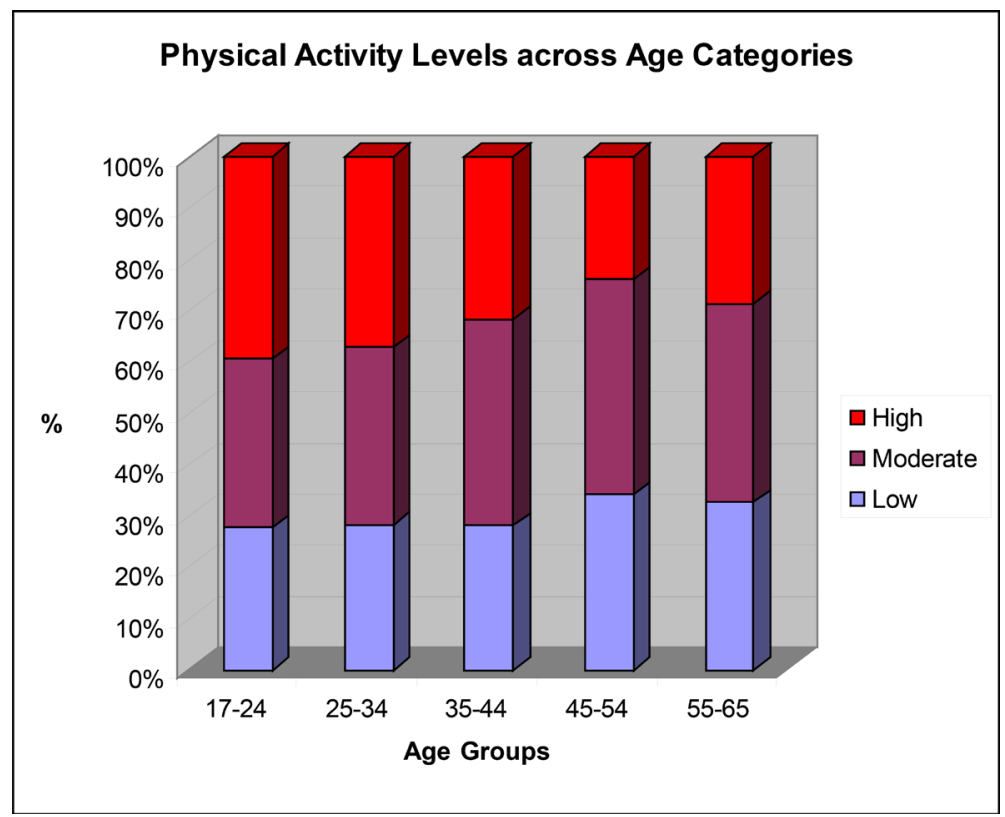

Figure 2. Percentages of the 3 categorical PA levels across age categories. 
in the obese group $(\mathrm{p}<0.05)$. Subjects with normal BMI were significantly more likely to be in high PA group ( $\mathrm{p}<0.05)$. Overweight subjects were more likely to be in a low or moderate PA level, when compared to those with normal BMI ( $\mathrm{p}$ $<0.05$ ). Almost half of those with high PA level had normal BMI and nearly $2 / 3$ of those with low PA level had BMI of $25 \mathrm{~kg} / \mathrm{m}^{2}$ or higher.

Males were more likely to be in high PA level if they were single (51.1\%) than if they were married (29.4\%). Females, on the other hand, had statistically insignificant differences $(\mathrm{p}>0.4)$ between married and singles in the proportions at the various PA levels.

Among females, status of employment did not significantly affect the PA categorical level. Males, on the other hand, were more likely to be in the high PA category if they were students $(\mathrm{p}<0.05)$. Non-working males were least likely to be in high PA categorical level $(\mathrm{p}<0.05)$. As PA at work increases, the proportion in the high PA category increases and that in the low PA category decreases $(\mathrm{p}<0.05)$. PA at work had no significant effect on the proportion of subjects at the moderate PA category. Interestingly, 35.2\% of those whose work involves no or low PA still managed to reach a moderate PA category (obviously due to their PA outside of the work-related PA domain), and another 30.8\% achieved the high PA category. Nevertheless, subjects whose PA profile at work involved vigorous activity were significantly less likely to be in low categorical PA level (11.6\%), and more than half of them were in high PA category. This was especially the case among males, where only $8.2 \%$ of males doing vigorous activity at work were in the low PA category.

\subsection{Regular Exercise}

The data derived from KPAQ recall showed that $47.3 \%$ reported regular exercise. The proportion of males who regularly exercised (58.1\%) was higher than that in females $(39.8 \%)(\mathrm{p}<0.001)$. The proportion of regular exercisers decreases from $68 \%$ to $40 \%$ in males and from $57 \%$ to $30 \%$ in females from ages $17-24$ to ages $35-44$.

The percentage of subjects exercising regularly is significantly higher $(\mathrm{P}<$ 0.05) in singles compared to married subjects, and in students compared to workers or no workers. Those whose work involved moderate or vigorous PA reported regular exercise more than $55 \%$ of the time. Regular exercisers were more likely to be in normal range of BMI than non-exercisers, while almost $2 / 3$ of the obese were non-regular exercisers.

Regular exercisers are more likely $(\mathrm{p}<0.005)$ to be in higher categorical PA level. Among males, $85.2 \%$ of those with high PA level were regular exercisers; this percentage was $54.6 \%$ among females, and $69 \%$ overall.

Figure 3 shows the percent of subjects practicing each of the types of exercises reported by regularly exercising individuals. Walking is most popular (74.3\%), followed by soccer $(26.8 \%)$. Significant differences between males and females are noted; more females reported walking and aerobics, while males reported 
other types of exercise such as soccer significantly more than females.

\subsection{NCD Incidence}

Incidence of health conditions across age categories are shown in Figure 4. Percentage for all health conditions increase with age for males and females. The occurrence of disease states was significantly higher in females than males with regard to hypertension, osteoporosis and cancer (all 8 subjects reporting cancer were females). Larger percentage of females (48.6\%) perceived themselves as being overweight or obese than males did $(34.5 \%)(\mathrm{p}<0.05)$.

Low daily PA is a health condition considered to contribute to non-communicable diseases (and a risk factor thereof). Its role can be appreciated when comparing the percentages of these health conditions across categorized ages. The percentage of subjects in the low categorical PA level (from IPAQ) is high (29.4\%) similar in

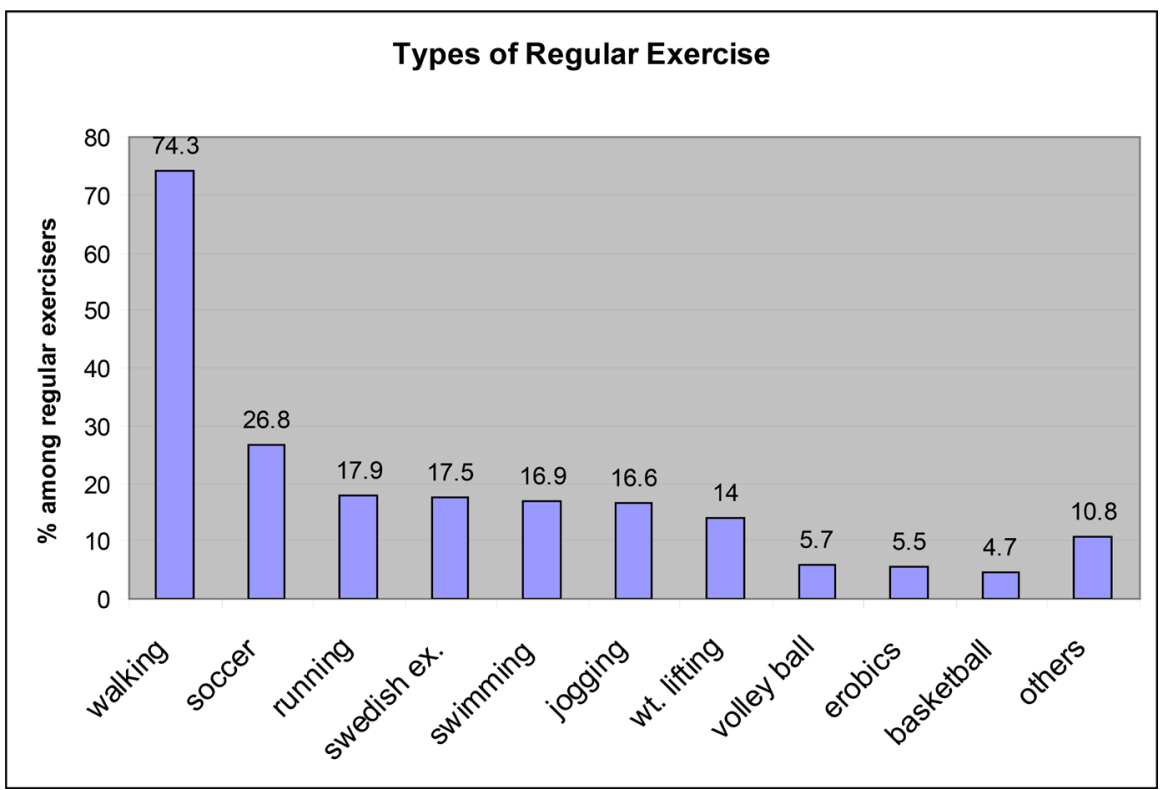

Figure 3. Percentages of types of exercise reported by subjects who reported regular exercise.

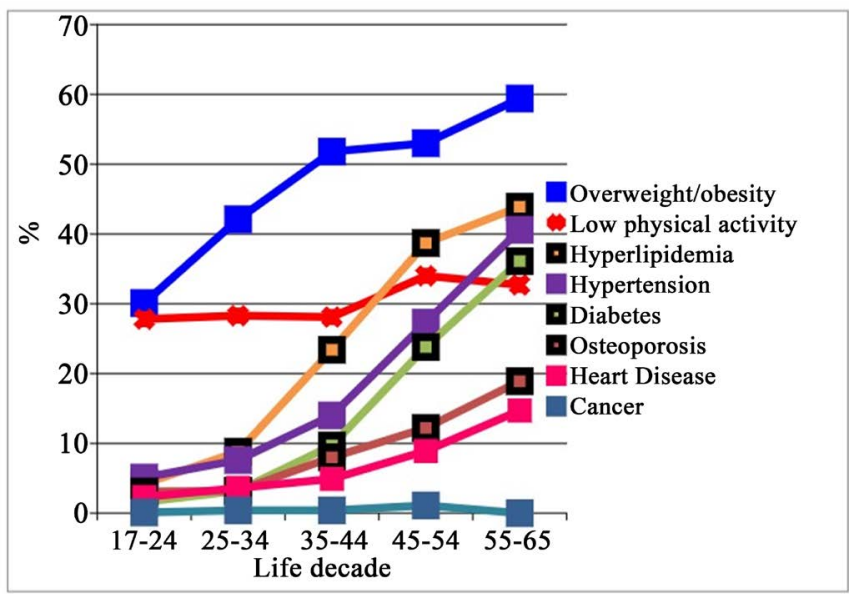

Figure 4. Prevalence of pathologies by decade of life. 
prevalence to overweight/obesity (30\%). At older age categories, prevalence of low PA is surpassed by rising incidences of hyperlipidemia, hypertension, and diabetes.

Incidence of overweight and obesity increased from 30 to more than 55\% from the second to the sixth decade of life. Hyperlipidemias increased in incidence from $8 \%$ to $45 \%$ of the sample from the $3^{\mathrm{d}}$ to the sixth decade of age. Hypertension increased from the $4^{\text {th }}$ to the sixth decades of life to affect from 14 to $40 \%$ of the sample. Diabetes incidence increased from $10 \%$ to $36 \%$ of the studied population, from the second to the sixth decades of life. Incidence of osteoporosis and Heart Disease only increased markedly, affecting from 12 to 18 and from 9 to $15 \%$ of the sample, respectively, between the $5^{\text {th }}$ and $6^{\text {th }}$ decades of life (Figure 4).

\subsection{Yearly Increments in NCD Incidence}

The yearly rates of increment in the incidence of these diseases were calculated and plotted as a function of the decades of age (Figure 5). In the second decade of life, overweight/obesity was dominant with an increase incidence of $1.2 \%$ year, decreasing to $1 \% /$ year in the $3^{\mathrm{d}}$ decade and to $0.1 \% /$ year in the $4^{\text {th }}$ decade (denoting little change in incidence), rebounding again to $0.6 \% /$ year in the fifth decade of life.

Increments in the yearly incidence rates of hyperlipidemia were 5 -fold from $0.3 \% /$ year to $1.5 \%$ /year from the second to the third decades of life, decreasing slightly to $1.4 \% /$ year at the fourth decade and to $0.5 \%$ year in the $5^{\text {th }}$ decade of life.

The increments in the incidence of diabetes reached a peak (1.4\% per year) in the $4^{\text {th }}$ decade from rates of $0.2 \%$ per year in the twenties, and $0.6 \% / y e a r$ in the thirties. The rate of increase in the incidence of diabetes, although high, slowed slightly in the $5^{\text {th }}$ decade to $1.2 \% /$ year.

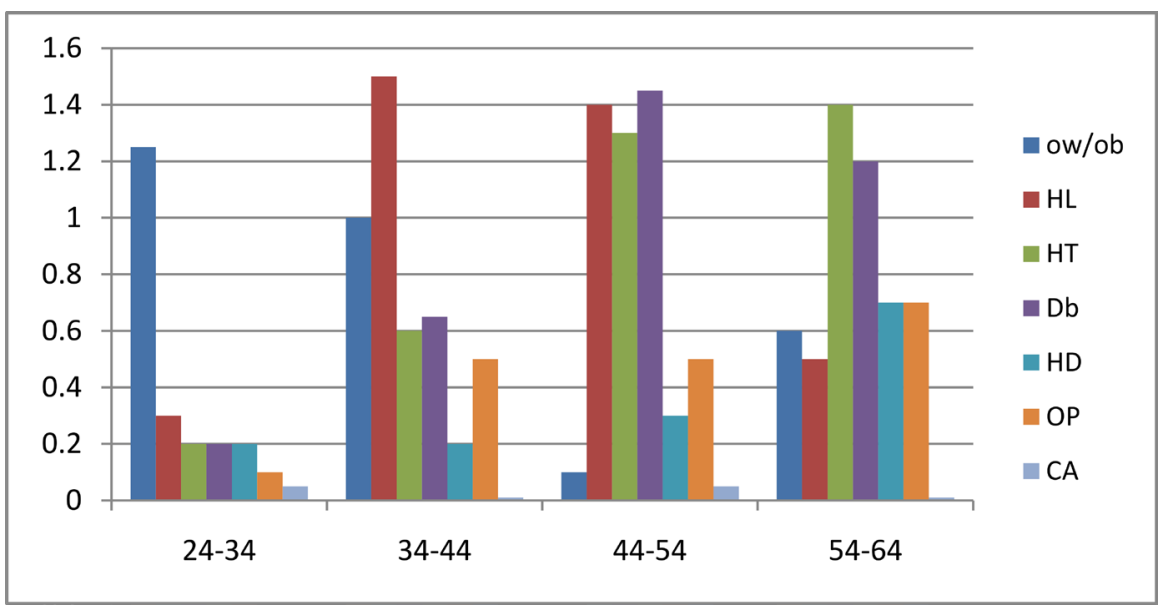

Ow/ob overweight and obesity, HL hyperlipidemia, HT hypertension, Db Diabetes, HD Heat Disease, OP Osteoporosis, CA cancers.

Figure 5. Yearly change in $\%$ incidence of pathologies at each decade of life. 
By contrast the rate of increase in the incidence of Hypertension was progressively higher at all ages from 0.2 to 0.6 , to $1.3 \%$ and $1.4 \%$ per year, at the $2 \mathrm{~d}, 3 \mathrm{~d}$, $4^{\text {th }}$ and $5^{\text {th }}$ decades of life, respectively.

Osteoporosis had spurts in its incidence in the $3 \mathrm{~d}$ decade (0.5\%/year), likely related to pregnancy and lactation and again in the $5^{\text {th }}$ decade $(0.7 \% /$ year $)$, probably, related to menopause.

The rate of increase in the incidence of heart disease was from $0.2 \% /$ year in the $2 \mathrm{~d}$ and $3 \mathrm{~d}$ decades to $0.3 \% / y e a r$ in the $4^{\text {th }}$ and to more than $0.7 \% / y e a r$ in the fifth decade of life.

Prevalence of low PA in the second decade of life is similar to that of obesity. However low PA prevalence did not change from the second to the third decade of age while obesity increases markedly. In the fourth decade, prevalence of low PA increases but obesity incidence is unchanged. In the fifth decade low PA prevalence does not increase any further but obesity prevalence again increases. These observations suggest that changes in EE for PA are not associated with the observed changes in the prevalence of obesity/overweight with age, which instead, are likely due to variations in energy intake (Figure 6).

\section{Discussion}

\subsection{PA of Kuwaiti Adults in This Sample}

These PA data are for subjects that reported participating in bouts of PA during the week and do not consider those that did not report any PA which was $15 \%$

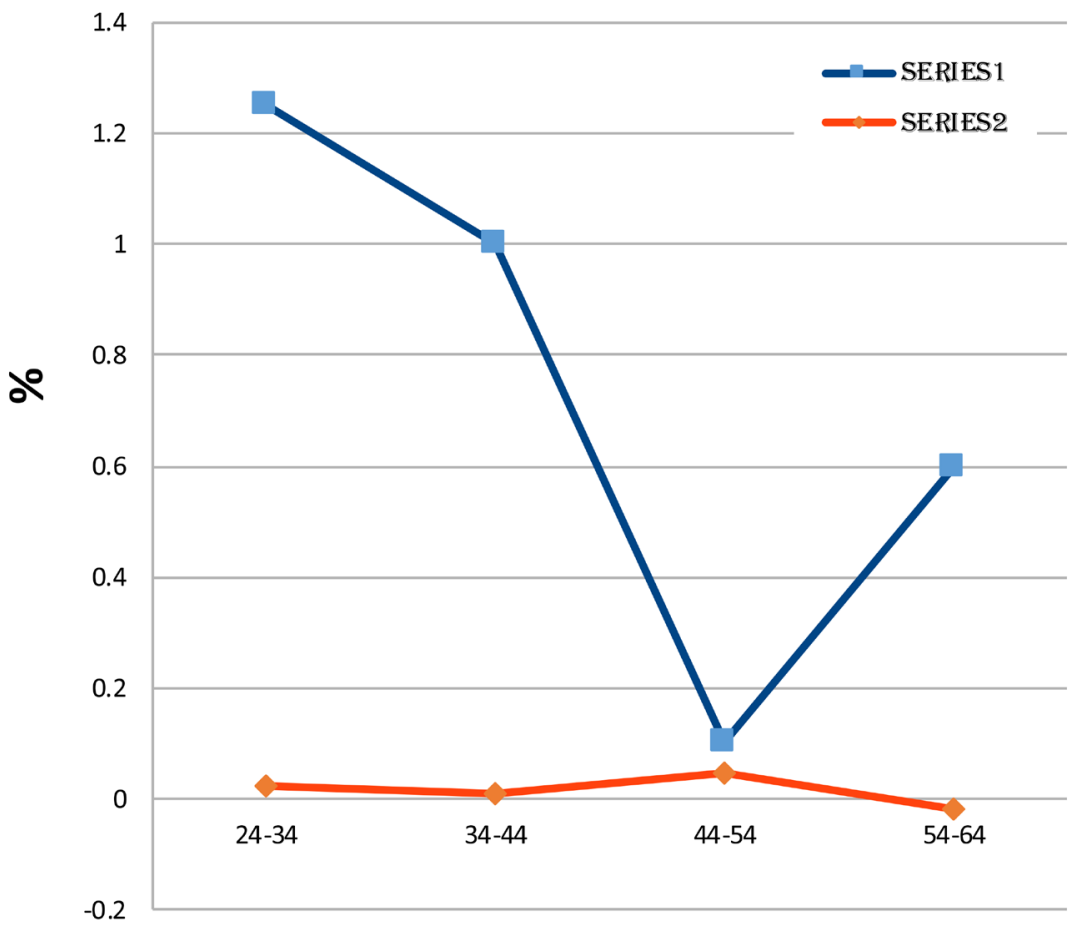

Life Decade

Figure 6. Yearly changes in \% incidence of Overweight/Obesity (blue) and of Low PA (red) by decade of life. 
(no walking), 29.3\% (no Moderate PA) and 46\% (no Vigorous PA) of the total sample. The overall categorically weighed EE PA estimate was $1280 \mathrm{MET} . \mathrm{min} / \mathrm{w}$, (see Table 1) an activity level slightly higher than that of a moderately active subject doing $60 \mathrm{~min} /$ day, 5 days per week of moderate intensity activities (1200 MET.min/w). Indeed most (40\%) subjects in this sample were in the moderate PA category (Table 1). In females, median (1500) and mean (2500) PA in MET.min/w, differed markedly as they did in males (1800 and 3000), pointing to the abnormal distribution of the PA data due to the high proportion of young subjects in the sample.

Inclusion of a relatively large group of young subjects, influenced the estimates of PA EE for this whole group of Kuwaiti subjects. The 17 - 24 years old group was $38 \%$ of the total, included many highly PA subjects and is much larger than the $12.5 \%$ it represents in the corresponding Kuwait population. The sample group was more educated, more were from the Jahra area and fewer from the Ahmadi and Farwania areas than those in the corresponding Kuwait census population. The sampled group had more females than the corresponding Kuwaiti population. These sampling issues reflect the difficulty of random sampling when recruiting volunteers.

Our study indicated that $48.5 \%$ of subjects walked for 150 minutes or more per week. It is relevant that walking is the most popular PA as reported by $74.3 \%$ of those who do regular exercise. Corresponding data from outside Kuwait are variable: $33.3 \%$ of Saudis aged 15 years and above walked for 150 minutes or more per week [23]. In the USA $33.7 \%$ of population were regular walkers vs. $20.7 \%$ that never walk [27]. In our study, never walkers comprised $15.4 \%$ only.

A study in Sweden using the short form of IPAQ [22] found that the percentage of the sample falling in low, moderate, and high categorical PA levels were $37 \%, 37 \%$, and 26\%; respectively. The high and the low PA groups were smaller than in our Kuwaiti sample.

Al-Hazzaa [23] described the PA profile of Saudi adults in Riyadh using the official Arabic short form of IPAQ intended for use in telephone interview [23]. The final sample size was 1064 . Based on the three activity categories established by IPAQ, results showed that $40.6 \%$ of Saudis were inactive (low PA category), more than in this Kuwait sample, $34.3 \%$ were minimally active (now known as moderate PA level) and only $25.1 \%$ met so-called health-enhancing levels of PA (i.e. high PA level), smaller than here described (33\%) for this Kuwait sample.

In our Kuwaiti sample, about $30 \%$ were insufficiently active, $37 \%$ were moderately active and $33 \%$ reached a high level of PA. The \% of subjects with low level of PA increased after 45 years of age and the high PA group became smaller with age as the chances for development of chronic diseases increase. The weighted average categorical PA level in this Kuwaiti sample is higher than in previous reports due to effects of WHO promotional campaigns for PA and Health directed and organized by Dr Jasem Ramadan Al-Kandari [28], active for more than 10 years with the Kuwait National plan for PA and Health [28], and 
to the high proportion of young people in the sample.

An interview-format IPAQ was used to assess PA and its relationship with self-rated health status in the 15 member states of the European Union (collected by the Eurobarometer project) [29]. On a descriptive level, results indicated positive relationships between PA level and self-rated health status across population subgroups by age, gender, income, and educational level. Also in a multivariate analysis, PA level was significantly related to better self-rated health status. We discuss below the incidence of various chronic diseases in Kuwait.

\subsection{Non-Communicable Diseases in Kuwait}

According to the Kuwait Institute for Health measure and evaluation, KIHME in 2016 among about 4.5 million Kuwaitis (and about 3.5 million expats) there were 11,000 deaths and NCD accounted for $72 \%$ of all deaths in the country as follows: $41 \%$ cardiovascular disease and stroke, $15 \%$ Cancers of all types, $13 \%$ accidents-injuries, $10 \%$ other NCD, 3\% diabetes, 3\% respiratory chronic disease, $15 \%$ genetic, perinatal, nutritional and communicable diseases. The probability for premature death in those below 70 years was $17 \%$, and there was a $2 \%$ suicide rate.

Among explored disease risk factors Physical inactivity was paramount at $65 \%$ while Obesity was $37 \%$ in those above 18 years and $23 \%$ in those between $10-18$ years of age, Tobacco smoking was at $24 \%$, salt intake averaged $10 \mathrm{~g} / \mathrm{day}$, hypertension was detected in $18 \%$, and high blood glucose in $15 \%$ of those above 18 years of age.

A 2006 survey of 2487 people in an urban and a more rural Kuwait governorates, including males and females 50 years and older indicated very high incidence of $53 \%$ for hypertension, $51 \%$ for diabetes, and $18 \%$ for heart disease, respectively. This represented a sharp increase in incidence compared to those observed 10 years earlier, indicating active progressive disease processes [30].

A more recent study concluded that current NCD research in Kuwait is not aligned with the disease burden or health priorities of the country [31].

\subsection{Age and NCD in the Current Study}

Although our current cross sectional data (Figure 4 and Figure 5) are suggestive of a sequence of events, initiated by overweight and obesity as early as in the $2 \mathrm{~d}$ decade of life, followed by hyperlipidemia in the third decade, diabetes and hypertension in the $4^{\text {th }}$, and osteoporosis and heart disease in the fifth decade of life, this sequence can only be confirmed after a true longitudinal follow up of a sufficient number of patients. The mechanisms leading to hyperlipidemia and hypertension in obesity are multiple and complex. Development of diabetes and heart disease is also multifactorial.

Pharmaceutical [32], surgical [33], as well as dietary interventions [34] to minimize overweight and hyperlipidemia should be evaluated, alone or in combination. Increases in PA may potentiate the responses to these treatments. 
The observed changes in events with age is suggestive of the points at which specific interventions may be most useful in preventing or reducing specific types of chronic diseases. Treatment of overweight and obesity should start at the second decade of life. Treatment of hyperlipidemia, should start early in the $3^{\mathrm{d}}$ decade of life. It remains to be seen if such early intervention results in significant prevention or delays in the development of hypertension, diabetes and heart disease.

\subsection{Limitations}

A major limitation of this study is its cross sectional nature, such that what is happening to 17 - 24 years old youth is not what the $54-65$ years old in the group lived through. A second major limitation is the nature of the sample which included a group of young subjects $17-24$ years of age, larger than in the corresponding \% in the Kuwait census population. However, the data and conclusion obtained in subjects 25 - 65 years old, which reflect well the age distribution in the target population of Kuwait, reaffirm most findings except for the relatively high levels of PA described by the medians and averages of the entire studied group.

\subsection{PA and Obesity}

The current observations confirmed the association of low PA with high BMI. They also indicate that changes in the incidence of low EE did not account for the observed changes in prevalence of obesity/overweight with age which instead are likely due to changes in energy intake in the presence of groups with continuously insufficient PA. Thus obesity is a cause of low PA rather than a consequence of it. Sitting time did not show any association with the incidence of the chronic diseases investigated in this Kuwaiti sample.

\section{Acknowledgements}

We acknowledge financial support by the Dept. of Physiology, Kuwait University.

This study was part of N. Garashi PhD dissertation directed by Dr Jasem Ramadan Al-Kandari at Kuwait University.

\section{Conflicts of Interest}

The authors declare no conflicts of interest regarding the publication of this paper.

\section{References}

[1] Garashi, N., Kandari, J., Ainsworth, B. and Barac-Nieto, M. (2020) Weekly Physical Activity from IPAQ (Arabic) Recalls and from IDEEA Activity Meters. Health, 12 598-611. https://doi.org/10.4236/health.2020.126045

[2] Pedersen, B.K. and Saltin, B. (2006) Evidence for Prescribing Exercise as Therapy in Chronic Disease. Scandinavian Journal of Medicine and Science in Sports, 16, 3-63. https://doi.org/10.1111/j.1600-0838.2006.00520.x 
[3] Bull, F. (2004) Comparative Quantification of Health Risks: Global and Regional Burden of Disease Attributable to Selected Major Risk Factors. World Health Organization (WHO), Geneva.

[4] Caspersen, C.J., Pereira, M.A. and Curran, K.M. (2000) Changes in Physical Activity Patterns in the United States, by Sex and Cross-Sectional Age. Medicine and Science in Sports and Exercise, 32, 1601-1609. https://doi.org/10.1097/00005768-200009000-00013

[5] Hegde, S.M. and Solomon, S.D. (2015) Influence of Physical Activity on Hypertension and Cardiac Structure and Function. Current Hypertension Reports, 17, Article No. 77. https://doi.org/10.1007/s11906-015-0588-3

[6] Jakicic, J.M. and Davis, K.K. (2011) Obesity and Physical Activity. Psychiatric Clinics of North America, 34, 829-840. https://doi.org/10.1016/j.psc.2011.08.009

[7] Cox, C.E. (2017) Role of Physical Activity for Weight Loss and Weight Maintenance. Diabetes Spectrum, 30, 157-160. https://doi.org/10.2337/ds17-0013

[8] Koopman, R.J., Mainous, A.G., Diaz, V.A. and Geesey, M.E. (2005) Changes in Age at Diagnosis of Type 2 Diabetes Mellitus in the United States, 1988 to 2000. The Annals of Family Medicine, 3, 60-63. https://doi.org/10.1370/afm.214

[9] Tuomilehto, J., Lindström, J., Eriksson, J.G., et al. (2001) Finnish Diabetes Prevention Study Group. Prevention of Type 2 Diabetes Mellitus by Changes in Lifestyle among Subjects with Impaired Glucose Tolerance. New England Journal of Medicine, 344, 1343-1350. https://doi.org/10.1056/NEJM200105033441801

[10] Folsom, A.R., Kushi, L.H. and Hong, C.P. (2000) Physical Activity and Incident Diabetes Mellitus in Post-Menopausal Women. American Journal of Public Health, 90, 134-138. https://doi.org/10.2105/AJPH.90.1.134

[11] Okada, K., Hayashi, T., Tsumura, K., Suematsu, C., Endo, G. and Fujii, S. (2000) Leisure-Time Physical Activity at Weekends and the Risk of Type 2 Diabetes Mellitus in Japanese Men: The Osaka Health Survey. Diabetic Medicine, 17, 53-58. https://doi.org/10.1046/j.1464-5491.2000.00229.x

[12] Thune, I. and Furberg, A.-S. (2001) Physical Activity and Cancer Risk: Dose-Response and Cancer, All Sites and Site-Specific. Medicine and Science in Sports and Exercise, 33, S530-S550. https://doi.org/10.1097/00005768-200106001-00025

[13] Cleveland, R.J., Eng, S.M., Stevens, J., et al. (2012) Influence of Prediagnostic Recreational Physical Activity on Survival from Breast Cancer. European Journal of Cancer Prevention, 21, 46-54. https://doi.org/10.1097/CEJ.0b013e3283498dd4

[14] Orsini, N., Bellocco, R., Bottai, M., et al. (2009) A Prospective Study of Lifetime Physical Activity and Prostate Cancer Incidence and Mortality. British Journal of Cancer, 101, 1932-1938. https://doi.org/10.1038/sj.bjc.6605404

[15] Brill, P., Macera, C.A., Davis, D.R., Blair, S.N. and Gordon, N. (2000) Muscular Strength and Physical Function. Medicine and Science in Sports and Exercise, 32, 412-416. https://doi.org/10.1097/00005768-200002000-00023

[16] Pinheiro, M.B., Oliveira, J., Bauman, A., et al. (2020) Evidence on Physical Activity and Osteoporosis Prevention for People Aged 65+ Years: A Systematic Review to Inform the WHO Guidelines on Physical Activity and Sedentary Behaviour. International Journal of Behavioral Nutrition and Physical Activity, 17, Article No. 150. https://doi.org/10.1186/s12966-020-01040-4

[17] Helasoja, V., Prättälä, R., Dregval, L., Pudule, I. and Kasmel, A. (2002) Late Response and Item Nonresponse in the Finbalt Health Monitor Survey. European Journal of Public Health, 12, 117-123. https://doi.org/10.1093/eurpub/12.2.117

[18] Ainsworth, B., Macera, C.A., Jones, D.A., et al. (2006) Comparison of the 2001 
BRFSS and the IPAQ Physical Activity Questionnaires. Medicine \& Science in Sports \& Exercise, 38, 1584-1592. https://doi.org/10.1249/01.mss.0000229457.73333.9a

[19] Craig, C., Russell, S.J. and Cameron, C. (2002) Reliability and Validity of Canada's Physical Activity Monitor for Assessing Trends. Medicine \& Science in Sports \& Exercise, 34, 1462-1467. https://doi.org/10.1097/00005768-200209000-00010

[20] Craig, C., Marshall, A.L., Sjöström, M., et al. (2003) International Physical Activity Questionnaire: 12-Country Reliability and Validity. Medicine \& Science in Sports \& Exercise, 35, 1381-1395. https://doi.org/10.1249/01.MSS.0000078924.61453.FB

[21] Bull, F.C., Maslin, T.S. and Armstrong, T. (2009) Global Physical Activity Questionnaire (GPAQ): Nine Country Reliability and Validity Study. Journal of Physical Activity and Health, 6, 790-804. https://doi.org/10.1123/jpah.6.6.790

[22] Bergman, P., Grjibovski, A.M., Hagströmer, M., Sallis, J.F. and Sjöström, M. (2009) The Association between Health Enhancing Physical Activity and Neighborhood Environment among Swedish Adults-A Population-Based Cross-Sectional Study. International Journal of Behavioral Nutrition and Physical Activity, 6, Article No. 8. https://doi.org/10.1186/1479-5868-6-8

[23] Al-Hazzaa, H. (2007) Health-Enhancing Physical Activity among Saudi Adults Using the International Physical Activity Questionnaire (IPAQ). Public Health Nutrition, 10, 59-64. https://doi.org/10.1017/S1368980007184299

[24] Carlson, S.A., Adams, E.K., Yang, Z. and Fulton, J.E. (2018) Percentage of Deaths Associated With Inadequate Physical Activity in the United States. Preventing Chronic Disease, 15, 170354. https://doi.org/10.5888/pcd18.170354

[25] Breda, J., Jakovljevic, J., Rathmes, G., Mendes, R., Fontaine, O., Hollmann, S., Rütten, A., Gelius, P., Kahlmeier, S. and Galeaa, G. (2018) Promoting Health-Enhancing Physical Activity in Europe: Current State of Surveillance, Policy Development and Implementation. Health Policy, 122, 519-527.

https://doi.org/10.1016/j.healthpol.2018.01.015

[26] Althoff, T., Sosič, R., Hicks, J.L., King, A.C., Delp, S.L. and Leskovec, J. (2017) Large-Scale Physical Activity Data Reveal Worldwide Activity Inequality. Nature, 547, 336-339. https://doi.org/10.1038/nature23018

[27] Eyler, A., Brownson, R.C., Bacak, S.J. and Housemann, R.A. (2003) The Epidemiology of Walking for Physical Activity in the United States. Medicine and Science in Sports and Exercise, 35, 1529-1536.

https://doi.org/10.1249/01.MSS.0000084622.39122.0C

[28] World Health Organization (2004) WHO RESOLUTION. Global Strategy on Diet, Physical Activity and Health.

http://www.who.int/dietphysicalactivity/strategy/eb11344/strategy english web.pdf

[29] Abu-Omar, K., Rütten, A. and Robine, J.-M. (2004) Self-Rated Health and Physical Activity in the European Union. Social and Preventive Medicine, 49, 235-242. https://doi.org/10.1007/s00038-004-3107-x

[30] Shah, N.M., Behbehani, J. and Shah, M.A. (2010) Prevalence and Correlates of Major Chronic Illnesses among Older Kuwaiti Nationals in Two Governorates. Medical Principles and Practice, 19, 105-112. https://doi.org/10.1159/000273069

[31] Badr, H., Maktabi, M.A., Al-Kandari, M. and Sibai, A.M. (2019) Review of Non-Communicable Disease Research Activity in Kuwait: Where Is the Evidence for the Best Practice? Annals of Global Health, 85, 45.

https://doi.org/10.5334/aogh.2392

[32] Mehta, A., Marso, S.P. and Neeland, I.J. (2017) Liraglutide for Weight Management: 
A Critical Review of the Evidence. Obesity Science \& Practice, 3, 3-14. https://doi.org/10.1002/osp4.84

[33] Colquitt, J.L., Pickett, K., Loveman, E. and Frampton, G.K. (2014) Surgery for Weight Loss in Adults. Cochrane Database of Systematic Reviews Review, 8, CD003641. https://doi.org/10.1002/14651858.CD003641.pub4

[34] Koliaki, C., Spinos, T., Spinou, M., Brinia, M.-E., Mitsopoulou, D. and Katsilambros, N. (2018) Defining the Optimal Dietary Approach for Safe, Effective and Sustainable Weight Loss in Overweight and Obese Adults. Healthcare (BaseI), 6, 73. https://doi.org/10.3390/healthcare6030073 